Original Article

\title{
Curcumin attenuates renal ischemia reperfusion injury via JNK pathway with the involvement of p300/CBP-mediated histone acetylation
}

\author{
Lu Yang ${ }^{1, \#}$, Xiaoxiang Chen ${ }^{1, \#}$, Zirong Bi ${ }^{2}$, Jun Liao ${ }^{3}$, Weian Zhao ${ }^{1, *}$, and Wenqi Huang ${ }^{1, *}$ \\ Departments of ${ }^{1}$ Anesthesiology and ${ }^{2}$ Organ Transplantation, The First Affiliated Hospital of Sun Yat-sen University, Guangzhou 510080, ${ }^{3}$ Department of \\ Organ Transplantation, Zhujiang Hospital of Southern Medical University, Guangzhou 510000, P.R. China
}

\section{ARTICLE INFO}

Received February 15, 2021

Revised May 22, 2021

Accepted June 11, 2021

*Correspondence

Wenqi Huang

E-mail: Huangwq@mail.sysu.edu.cn

Weian Zhao

E-mail: zhaoweian5@mail.sysu.edu.cn

\section{Key Words}

Curcumin

Histone acetylation

MAP kinase signaling system

p300/CBP

Reperfusion Injury for Renal IRI

\#These authors contributed equally to this work.
ABSTRACT Apoptosis is proved responsible for renal damage during ischemia/reperfusion. The regulation for renal apoptosis induced by ischemia/reperfusion injury (IRI) has still been unclearly characterized to date. In the present study, we investigated the regulation of histone acetylation on IRI-induced renal apoptosis and the molecular mechanisms in rats with the application of curcumin possessing a variety of biological activities involving inhibition of apoptosis. Sprague-Dawley rats were randomized into four experimental groups (SHAM, IRI, curcumin, SP600125). Results showed that curcumin significantly decreased renal apoptosis and caspase-3/-9 expression and enhanced renal function in IRI rats. Treatment with curcumin in IRI rats also led to the decrease in expression of p300/cyclic AMP response element-binding protein (CBP) and activity of histone acetyltransferases (HATs). Reduced histone H3 lysine 9 (H3K9) acetylation was found near the promoter region of caspase-3/-9 after curcumin treatment. In a similar way, SP600125, an inhibitor of c-Jun N-terminal kinase (JNK), also attenuated renal apoptosis and enhanced renal function in IRI rats. In addition, SP600125 suppressed the binding level of p300/CBP and H3K9 acetylation near the promoter region of caspase-3/-9, and curcumin could inhibit JNK phosphorylation like SP600125. These results indicate that curcumin could attenuate renal IRI via JNK/p300/CBP-mediated anti-apoptosis signaling.

\section{INTRODUCTION}

Renal ischemia/reperfusion injury (IRI) is a common cause of early allograft dysfunction after kidney transplantation and represents an additional risk factor for the late renal allograft failure $[1,2]$. It also mainly contributes to acute kidney injury (AKI) which is a clinically common and heterogeneous disorder in hospitalized patients with high mortality, and can increase the risk for progression to end-stage renal disease [3]. Even a mild AKI may lead to a poor prognosis, and significantly increase a patient's in-hospital mortality, length of stay, and medical expenses [4]. The pathophysiology and pathogenesis of IRI remain complex and multifactorial. Experimental studies have proved that apoptosis of renal tubular epithelial cells plays a significant role in IRI $[5,6]$. Apoptosis results in loss of brush border, dilatated or damaged tubules, presence of intraluminal casts, and deterioration in renal function $[7,8]$. However, the molecular mechanism of renal tubular epithelial cell apoptosis in IRI still remains poorly understood.

Recent studies have revealed that epigenetic regulation, including histone acetylation, could be critically involved in the pathogenesis of renal IRI [9-11]. Histone acetylation on lysine residues could neutralize the positive charge, thereby destabilizing the histone-DNA interaction, subsequently changing the condensed (i) \$ This is an Open Access article distributed under the terms of the Creative Commons Attribution Non-Commercial License, which permits unrestricted non-commercial use, distribution, and reproduction in any medium, provided the original work is properly cited. Copyright @ Korean J Physiol Pharmacol, pISSN 1226-4512, elSSN 2093-3827
Author contributions: L.Y. and X.X.C. have contributed equally to this work. L.Y. and W.A.Z. designed the protocol of the study. L.Y. and X.X.C. performed the experiments. L.Y. wrote the manuscript. Z.R.B. and J.L. edited the manuscript. W.Q.H. supervised and coordinated the study. 
chromatin into an open, loosely packed chromatin structure, and consequently allowing recruitment of the activators for gene transcription $[12,13]$. Acetylation of histone was demonstrated to be involved in renal IRI [14]. Enhanced histone H3 acetylation caused by ischemia/reperfusion would damage renal function [15]. Besides, hyperacetylation of histone $\mathrm{H} 3$ could induce apoptosis in cancer cells [16-18]. These studies indicate that renal IRI induced apoptosis might be regulated by histone $\mathrm{H} 3$ acetylation. However, the mechanisms underlying the regulation of renal IRI on histone $\mathrm{H} 3$ acetylation remains unknown.

Curcumin, as a spice for culinary and food coloring purposes, has been widely used for centuries in indigenous medicine for daily treatment of various diseases [19]. It has been proved to be a specific inhibitor of the $\mathrm{p} 300 /$ cyclic AMP response elementbinding protein (CBP) family of histone acetyltransferases (HATs) [20]. Compelling evidence indicates that curcumin exerts a protective role against apoptosis in hepatocytes and cardiomyocytes [21,22]. To our knowledge, c-Jun N-terminal kinase (JNK) signaling pathway plays a critical role in apoptosis in renal IRI [23]. Furthermore, in vitro experiments showed that enhancement on activation of JNK signaling was correlated with elevated acetylation of histone $\mathrm{H} 3$ in hepatocytes and neurons [24]. In the present study, we investigated the role of curcumin on regulation of IRI-induced apoptosis. We found that curcumin inhibited renal apoptosis via suppression on p300/CBP induced H3K9 acetylation ( $\mathrm{acH} 3 \mathrm{~K} 9$ ) near the promoter region of caspase-3/-9 and then inhibited the expression of caspase-3/-9 in IRI rats. Furthermore, we used JNK inhibitor SP600125 to explore the effect of JNK signaling on HAT activity of p300/CBP and histone acetylation in renal IRI. Curcumin could inhibit JNK signaling in the similar way to SP600125. Our study indicates that curcumin could attenuate IRI induced renal apoptosis via inhibition of JNK/p300/CBP mediated regulation of caspase-3/-9 in IRI rats. Our data reveal that the inhibition of JNK/p300/CBP mediated histone acetylation could serve as a potential therapeutic path for renal IRI.

\section{METHODS}

\section{Animal modeling and grouping}

All experimental protocols were approved by the Institutional Ethics Committee of the First Affiliated Hospital of Sun Yat-sen University, China (no. 2017-103). And the study was conducted in accordance with the Basic \& Clinical Pharmacology \& Toxicology policy for experimental and clinical studies. A total of 40 adult male Sprague-Dawley rats weighing 180-200 g were used. Before experiment, rats were adaptively reared in separated ventilated cages under controlled temperature and humidity conditions in 12/12-h light/dark cycle with ad libitum access to standard rodent diet and water for one week. Right nephrectomy was performed in all rats to minimize its impact on the other kidney and maxi- mize the simulation modeling of kidney transplantation.

Briefly, all rats were anesthetized through intraperitoneal injection of pentobarbital ( $40 \mathrm{mg} / \mathrm{kg})$ and placed on a heating surgical pad. Following a midline laparotomy, the right renal vessels and right urethra were ligated, and right kidney was excised. The left renal pedicle was exposed and occluded for 45 min using an atraumatic arterial clamp (except for SHAM group). Afterwards, the clamps were removed to restore blood supply, and the wound was sutured in two layers and disinfected. Rats were randomly assigned into four groups of 8 animals each. Rats in SHAM group did not receive left renal ischemia and reperfusion. Rats in IRI group received left renal ischemia for $45 \mathrm{~min}$ and subsequent reperfusion for $24 \mathrm{~h}$. In IRI/Cur group, the rats received curcumin (Sigma-Aldrich, St. Louis, MO, USA) $200 \mathrm{mg} / \mathrm{kg} /$ day orally for 7 $\mathrm{d}$ prior to the left renal ischemia. Curcumin treatment protocol was performed with some modifications of previous procedure $[25,26]$. In IRI/SP group, the rats received intraperitoneal injection of JNK inhibitor SP600125 (MCE, Monmouth Junction, NJ, USA) at a dose of $15 \mathrm{mg} / \mathrm{kg}$ at $45 \mathrm{~min}$ before the left renal ischemia. SP600125 treatment protocol was performed with some modifications of previous procedure [27]. No adverse effects of SP600125 or curcumin alone on renal function were found under the applied drug dosage (data not shown). All rats were sacrificed under anesthesia $24 \mathrm{~h}$ after the initiation of reperfusion. Blood samples were collected via abdominal aorta, and left kidneys were harvested and immediately frozen in liquid nitrogen for subsequent operations.

\section{Histopathological investigation}

Tissues for histopathological investigation were harvested from the same site of a kidney. The kidney tissues were fixed with $4 \%$ paraformaldehyde for $24 \mathrm{~h}$ and then embedded in paraffin block and sliced into serial sections of $4 \mu \mathrm{m}$ thickness, followed by hematoxylin-eosin (H\&E) staining and periodic acid-Schiff (PAS) staining. At least 10 fields $(\times 200)$ for each slide were examined by a renal pathologist in a blinded fashion. A semi-quantitative scoring scale was made to evaluate renal tubule damage according to the extent of damage to the proximal convoluted tubule, interstitial edema and cellular casts. The scoring was performed by nephrologists based on H\&E staining and PAS staining. The extent of damage was scored as $0(<25 \%), 1(25 \%-50 \%), 2(50 \%-75 \%$ and 3 (> 75\%); the damage degree of proximal convoluted tubule was scored as 0 (no damage), 1 (brush border loss), 2 (flatten cells) and 3 (only the basilemma is seen); 0 score was given to the absence of interstitial edema, while 1 score to the presence of interstitial edema; the presence of protein casts alone was scored as 0 , while the presence of granular casts or cellular casts was scored as 1 . For TUNEL assay, In Situ Cell Death Detection Kit (Roche, Basel, Switzerland) was used to detect renal cell apoptosis. Paraffin embedded sections were dewaxed in xylene and rehydrated by passage through a graded ethanol series to tap water, and finally 
rinsed in phosphate-buffered saline. After digestion with proteinase $\mathrm{K}$, sections were incubated in a humidified chamber at $37^{\circ} \mathrm{C}$ for $60 \mathrm{~min}$ in dark for TUNEL staining. The sections were counterstained with DAPI (4,6-diamino-2-phenylindole) and then observed with an automatic fluorescence microscope (Nikon, Tokyo, Japan). Image J software (NIH Image, Bethesda, MD, USA) was used for cell counting, and apoptosis index was calculated as the ratio of the number of TUNEL-positive cells to the total number of DAPI-positive cells.

\section{Biochemical analysis}

Plasma was separated by centrifuging the blood samples obtained from abdominal aorta at 4,000 rpm for $10 \mathrm{~min}$. Plasma concentrations of serum creatinine (Scr) and blood urea nitrogen (BUN) were measured with HITACHI ${ }^{\circledR} 7020$ Biomedical Autoanalyzer (HITACHI, Tokyo, Japan).

\section{Western blotting}

Western blotting was performed as detailed in the previous study [28]. Proteins bound to the PDVF (polyvinylidene fluoride) membrane were incubated with primary antibodies against CBP (Abcam, Cambridge, UK), p300 (Abcam), GAPDH (Arigo, China), caspase-3 (CST, Boston, MA, USA), cleaved caspase-3 (CST), caspase-9 (CST), cleaved caspase-9 (CST), acH3 (CST), H3 (CST) and JNK (CST). Images were acquired using a G:BOX imaging system (Syngene, Cambridge, UK) and quantified using ImageJ software to obtain protein band intensity.

\section{Chromatin immunoprecipitation assay}

Chromatin immunoprecipitation (ChIP) assay was performed as described in the previous study [29] using a ChIP assay kit (Abcam). Protein-DNA complexes were incubated with a monoclonal antibody against acH3K9 (Abcam) at room temperature for $3 \mathrm{~h}$ for precipitation. Total column input served as a positive control, and non-immune immunoglobulin G (IgG) was applied as a negative control. The level of immunoprecipitated DNA was measured through quantitative real-time PCR (qRTPCR) using a KAPA SYBR FAST qPCR kit (Kapa Biosystems, Wilmington, MA, USA). For PCR, upstream primer pair of caspase-3 (1,000 bp) were: 5'TGAGGGAGCAAGAGTTAGG3' (DNA sense strand) and 5'ATTCCAGGGTCACAAAGG3' (DNA anti-sense strand); upstream primer pair of caspase-9 (1,000 bp) were: 5'GAGGCAAGGAAGAGCGAGTC3' (DNA sense strand) and 5'CCCACGGAGCACCTGAAT3' (DNA anti-sense strand). $\mathrm{Ct}$ value of each ChIP DNA fraction was normalized against the total input DNA fraction collected after release using the $\Delta \Delta \mathrm{CT}$ method.

\section{Histone acetyltransferase activity assay}

HAT activity assay was performed using the HAT Activity Fluorometric Assay Kit (BioVision, Milpitas, CA, USA). Nuclear extracts were isolated with the Nuclear/Cytosol Fractionation Kit (BioVision) as per the manufacturer's instructions. For sample preparation, $10 \mu \mathrm{l}$ of sample was obtained and mixed with HAT Assay Buffer to reach a volume of $50 \mu$ l. One well filled with $50 \mu \mathrm{l}$ HAT Assay Buffer was taken as background control. Then $4 \mu \mathrm{l}$ of HeLa Nuclear Extract was mixed with HAT Assay Buffer to reach a volume of $50 \mu$ l. The mixture was added into desired wells for positive control. Standard Curve Preparation and Reaction Mix were made as per the manufacturer's instructions. Then, $50 \mu \mathrm{l}$ of the reaction mix was added to each well containing the samples, background control, standards and positive control. Fluorescence at $\mathrm{Ex} / \mathrm{Em}=535 / 587 \mathrm{~nm}$ were measured in kinetic mode with a microplate reader (BioTek, Winooski, VT, USA) at $25^{\circ} \mathrm{C}$ for 60 min for the samples and background control, and the corresponding relative fluorescence unit (RFU) were obtained.

\section{Statistical analysis}

Data were expressed as mean \pm SD. All statistical analyses were performed using Statistical Product and Service Solutions (SPSS) 22.0 (IBM, Armonk, NY, USA) and the figures were processed using GraphPad Prism 6.0 software (GraphPad, San Diego, CA, USA). One-way ANOVA was used to analyze the differences among groups, while Fisher's Least Significant Difference test was used for pairwise comparison between groups. For all analyses, pvalues $<0.05$ was considered statistically significant.

\section{RESULTS}

\section{Curcumin could enhance renal function of IRI rats}

Scr and BUN levels were detected to indicate the renal func-
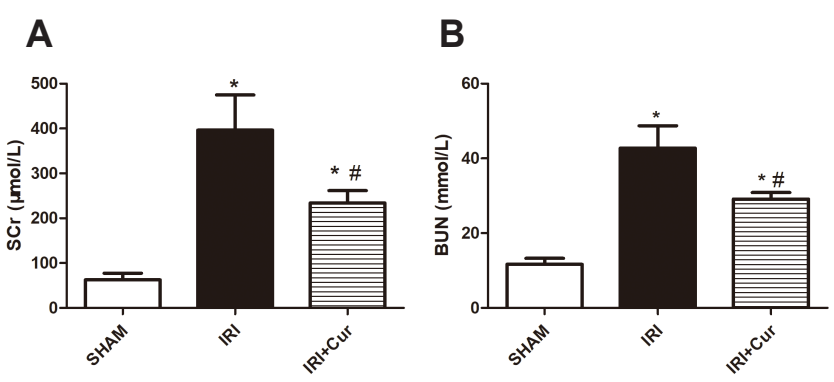

Fig. 1. Serum creatinine (Scr) and blood urea nitrogen (BUN) levels were used for assessing renal function. Scr (A) and BUN (B) levels in SHAM group, IRI group and IRI/Cur group. Statistical significance was determined by one-way ANOVA followed by Fisher's Least Significant Difference test. $\mathrm{n}=8$ per group. IRI, ischemia/reperfusion injury; Cur, curcumin. ${ }^{*} p<0.05$ vs. SHAM group; ${ }^{*} p<0.05$ vs. IRI group. 
tion of the rats. As shown in Fig. 1A, B, Scr and BUN levels were significantly elevated in IRI rats as compared with SHAM rats. Scr and BUN levels in IRI/Cur rats were significantly reduced as compared with IRI rats. H\&E staining and PAS staining were used to detect the renal morphological changes. As shown in Fig. 2A, IRI caused greater damage to renal proximal tubules mainly including brush border loss and flatten cells. Curcumin treatment could alleviate the damage to proximal tubules. The staining results of renal cortex were used for damage scoring via the modified semi-quantitative evaluation scale as described above. As shown in Fig. 2B, IRI rats received increased scores, but much lower scores after curcumin treatment.

\section{Curcumin inhibits renal tubule apoptosis of IRI rats}

TUNEL assay was used for in situ detection of apoptosis in renal tissue sections. As shown in Fig. 3, TUNEL-positive cells pervaded over renal cortex in IRI rats. Apoptosis index increased significantly in renal proximal tubules of IRI rats and decreased in IRI rats receiving curcumin treatment. We further detected apoptosis-associated proteins caspase- 3 and caspase- 9 via Western blotting. As shown in Fig. 4A, B, caspase-3/-9 in IRI rats were higher than those in SHAM rats in expression, while their expressions deceased in IRI rats receiving curcumin treatment. We further examined cleaved caspase- 3 and cleaved caspase- 9 which
A
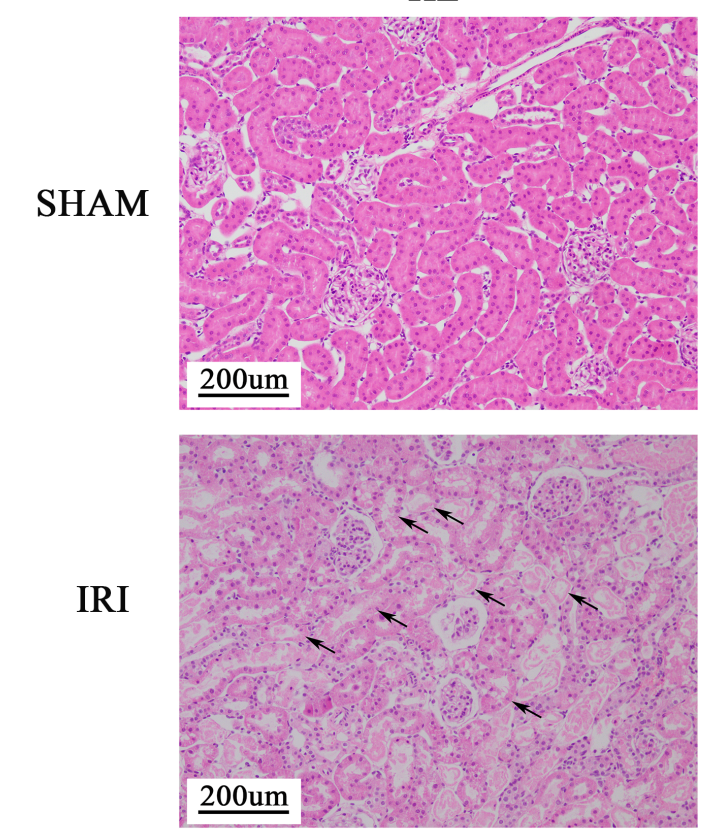

$\mathrm{IRI}+\mathrm{Cur}$

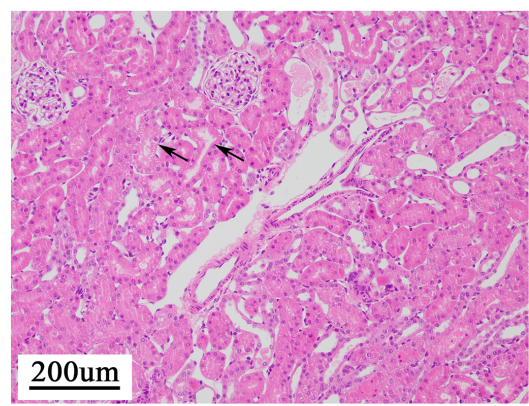

B

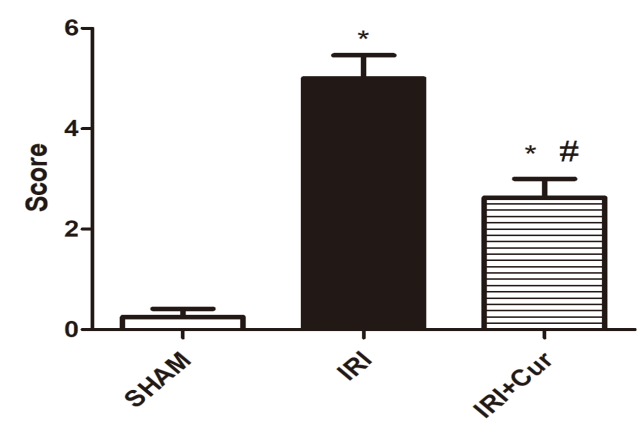

PAS
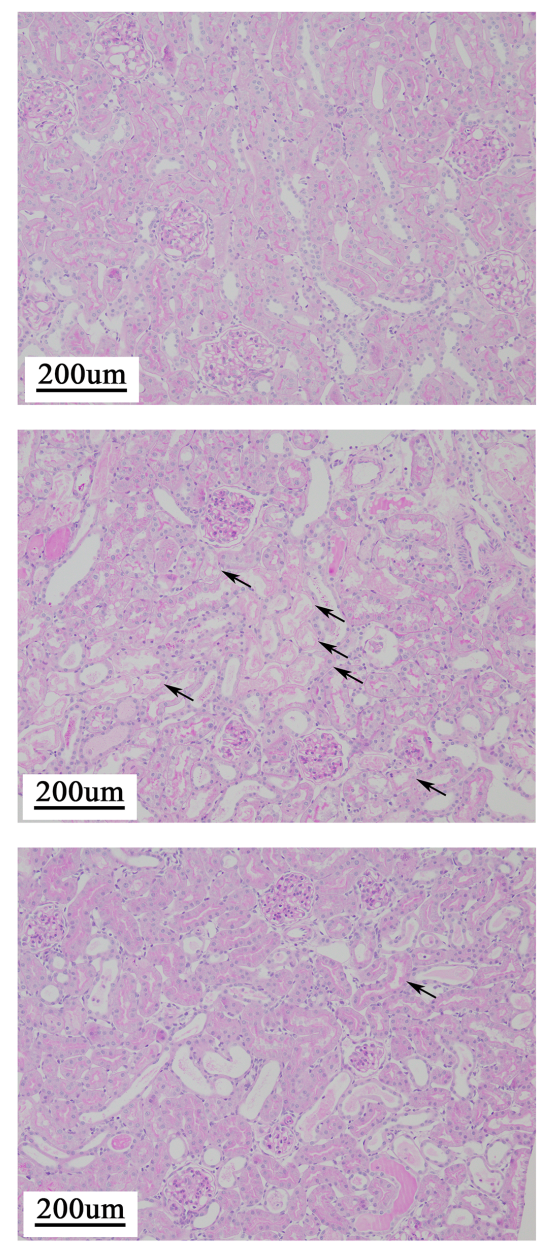

Fig. 2. Histopathological investigation in the presence or absence of curcumin treatment. (A) Representative images of renal tissue sections stained with H\&E and PAS. Black arrows indicate proximal tubule injuries mainly including brush border loss and flatten cells. (B) Semiquantitative scoring of renal injuries based on morphological changes in renal tissue sections stained with $\mathrm{H} \& \mathrm{E}$ and PAS. $n=8$ per group. IRI, ischemia/reperfusion injury; Cur, curcumin. ${ }^{*} p<0.05$ vs. SHAM group; $" \mathrm{p}<0.05$ vs. IRI group. 


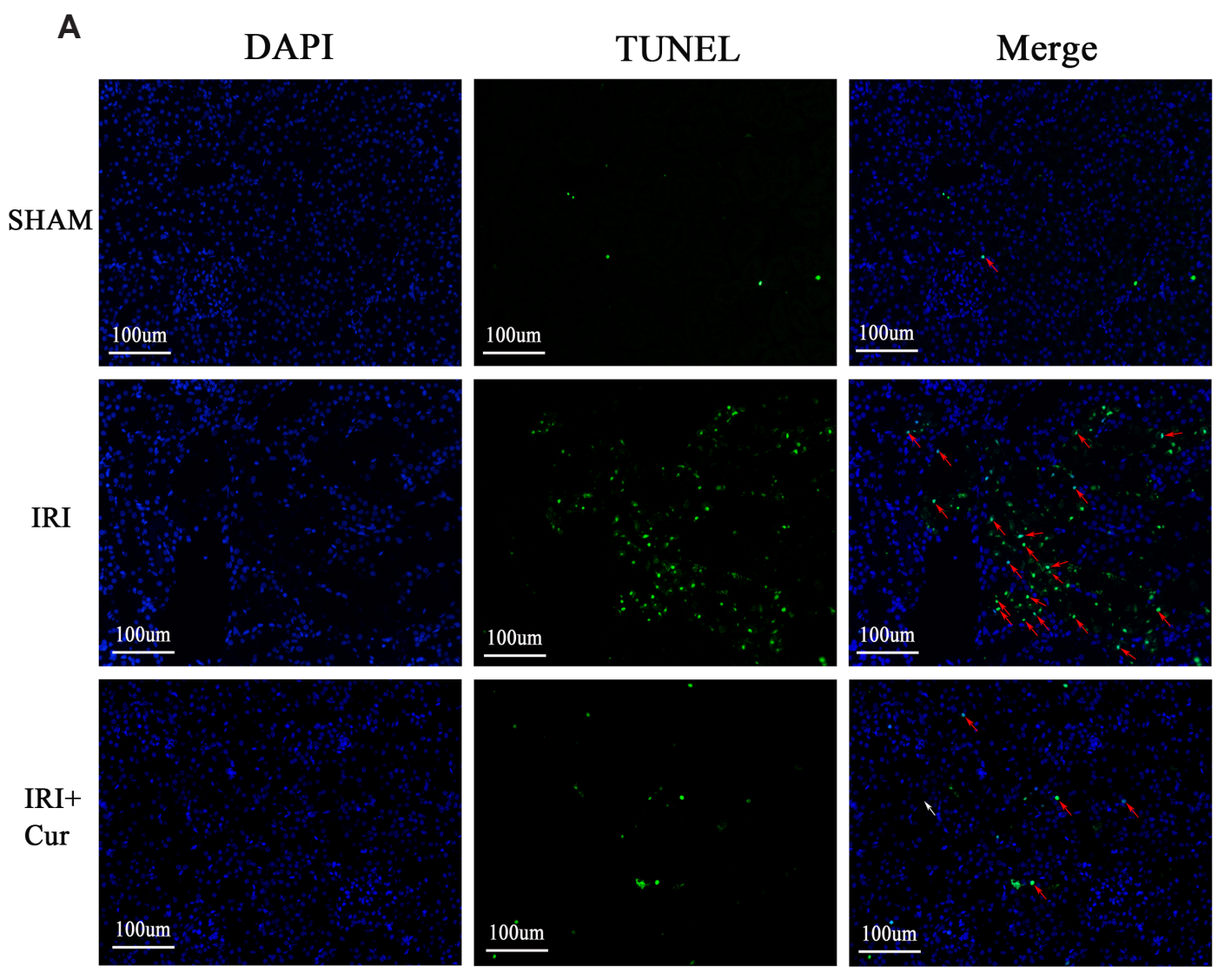

B

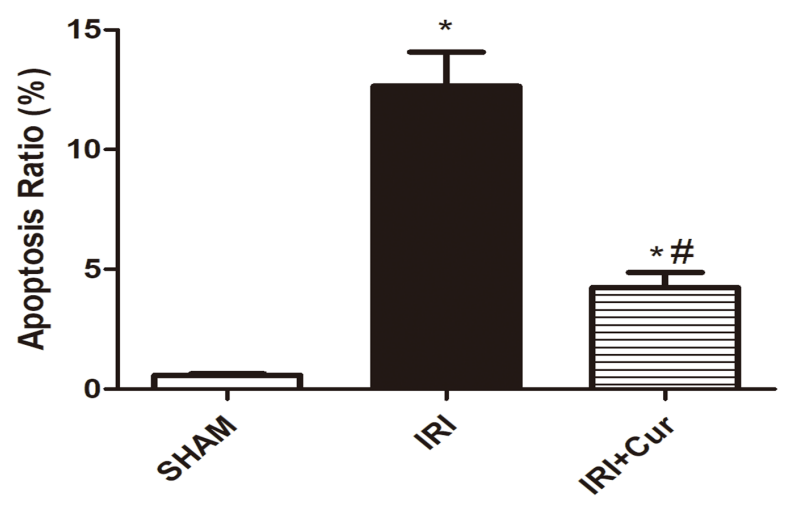

Fig. 3. TUNEL assay was used for in situ detection of apoptosis in renal tissue sections. (A) Representative images of renal cell apoptosis in SHAM group, IRI group and IRI/Cur group. Red arrows indicate TUNEL-positive cells. (B) Apoptosis indexes of above groups. $\mathrm{n}=8$ per group. IRI, ischemia/ reperfusion injury; Cur, curcumin. ${ }^{*} p<0.05$ vs. SHAM group; ${ }^{*} p<0.05$ vs. IRI group.

are small fragments extracted from the caspase and play a prominent role in caspase-dependent pathway for apoptosis. Cleaved caspase-3/-9 significantly increased in expression in IRI rats as compared with SHAM rats, while their expressions decreased in IRI rats receiving curcumin treatment.

\section{Curcumin could suppress caspase-3/-9 expression via inhibition of histone acetylation}

Histone H3K9 plays a critical role in apoptosis and its acetylation can regulate the cardiomyocytes apoptosis induced by alcohol by controlling caspase expression. In order to determine whether the changes of caspase-3/-9 in expression were associated with acetylated histone $\mathrm{H} 3 \mathrm{~K} 9$ ( $\mathrm{acH} 3 \mathrm{~K} 9$ ) near the promoter region 


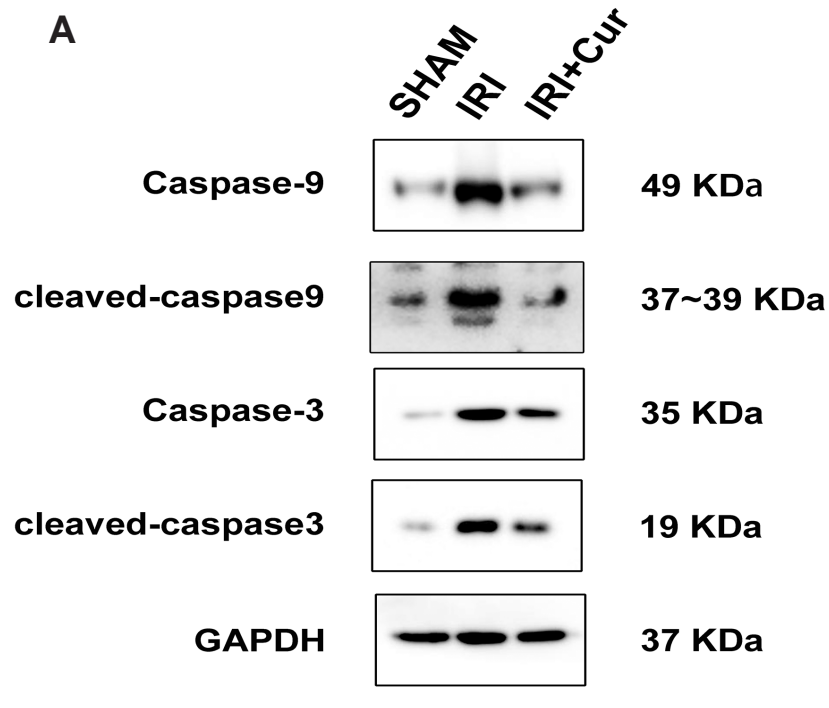

B

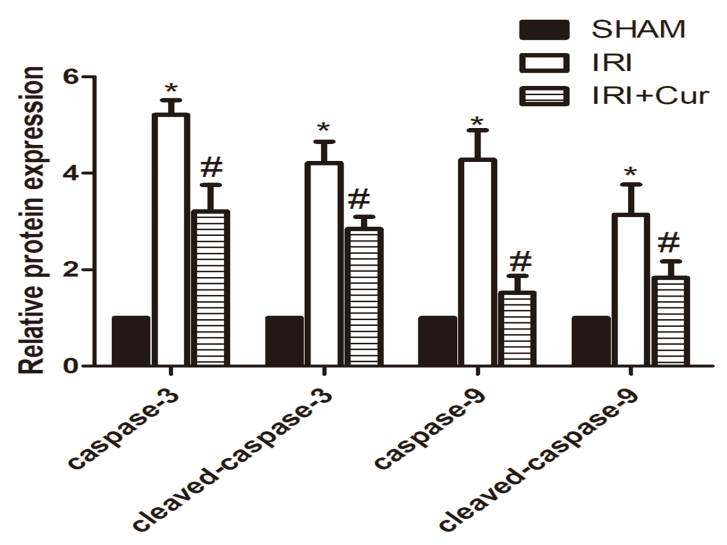

Fig. 4. Protein levels of caspae-3 and caspase-9 in IRI rats with or without curcumin treatment. (A) The protein levels of caspase-3/-9 and cleaved caspase-3/-9 in SHAM group, IRI group, and IRI/Cur group. (B) Quantification results of Western blotting. $n=8$ per group. IRI, ischemia/reperfusion injury; Cur, curcumin. ${ }^{*} \mathrm{p}<0.05$ vs. SHAM group; ${ }^{*} \mathrm{p}<$ 0.05 vs. IRI group.

of these genes, we assessed the acH3K9 level near the promoter region via ChIP assay. As shown in Fig. 5A, B, acH3K9 level increased near the promoter region of both caspase-3/-9 genes in IRI rats as compared with SHAM rats. While the acH3K9 level was reduced after curcumin treatment. To trace the cause of H3K9 acetylation, we detected the mRNA expression of HATs including p300/CBP, p300/CBP-associated factor and general control nonderepressible 5 which can acetylate H3K9. We found only p300/CBP increased in IRI rats as compared with SHAM rats (data not shown). This result was supported by Western blotting (Fig. 5E, F). Thus, we applied ChIP assay to find out whether p300/CBP caused the hyperacetylation of H3K9. As shown in Fig. $5 \mathrm{C}, \mathrm{D}$, the binding level of $\mathrm{p} 300 / \mathrm{CBP}$ to the promoter region of caspase-3/-9 increased in IRI rats as compared with SHAM rats, and relatively decreased in IRI rats receiving curcumin treatment.

\section{Curcumin regulates p300/CBP-mediated H3K9 acetylation via JNK signaling pathway}

In a similar way to curcumin, JNK inhibitor SP600125 was reported to suppress activation of caspase proteins in IRI rats. As shown in Fig. 6A, we confirmed the similar effect on the expression of caspase-3/-9 proteins in IRI rats receiving curcumin or SP600125 treatment. The similar effects on renal function (Supplementary Fig. 1), morphology and apoptotic index between SP600125 and curcumin (Supplementary Figs. 2 and 3) were shown in supplementary materials. Since JNK signaling was reported to regulate the expression and activity of p300/CBP, we further detected the relationship between curcumin and JNK pathway. As shown in Fig. 6B, similar to the effect of curcumin, SP600125 repressed the levels of CBP/p300 and acetylated Histone H3 in IRI rats. In a similar way to SP600125, curcumin could also inhibit phosphorylated JNK signaling in IRI rats (Fig. 6C). In addition, we detected acH3K9 level and the binding level of $\mathrm{CBP} / \mathrm{p} 300$ near the promoter region of caspase-3/-9. As shown in Fig. 7A, B, similar to the effect of curcumin, SP600125 repressed the enhanced acH3K9 in the promoter region of caspase-3/-9. SP600125 also inhibited the binding level of CBP/p300 to the promoter region of caspase-3/-9 (Fig. 7C, D). Furthermore, both SP600125 and curcumin inhibited HAT activity in IRI rats as shown in Fig. 7E.

\section{DISCUSSION}

Renal IRI is a common clinical phenomenon associated with kidney dysfunction and tissue damage. Its pathophysiology and pathogenesis are complex and multifactorial. Currently, apoptosis has been implicated in IRI but the mechanisms remain unclear. Unfortunately, no specific drugs have been found that could effectively prevent and treat renal IRI. Curcumin can mediate antiinflammatory, anti-oxidative, and anti-fibrosis effects and thus has therapeutic potential in IRI [30,31]. Mounting studies have confirmed that curcumin has protective effects against renal, hepatic and cardiac IRIs in rat due to its pharmacological activities [32-36]. In the present study, we found IRI could cause an increase in renal apoptosis and severe damage to renal tubules. Curcumin could alleviate proximal tubule damage, interstitial edema and cast formation in the treatment of renal IRI rats. In addition, Scr and BUN levels in IRI rats decreased after receiving curcumin treatment. These data indicate that apoptosis could be responsible for renal IRI and curcumin could protect renal function from IRI via its anti-apoptosis effect. Caspases are essential regulators for the initiation and execution of apoptosis. We in the present study found curcumin could repress the expression of cleaved caspase-3/-9 in IRI rats, which is consistent to the findings from Awad and El-Sharif [6]. All these data indicate that curcumin could alleviate IRI induced renal apoptosis via suppressing 
A

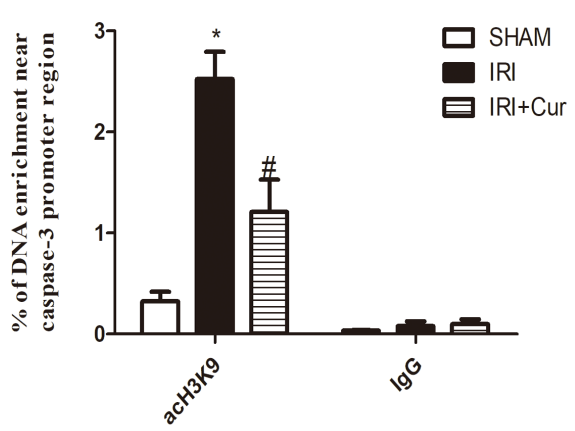

C

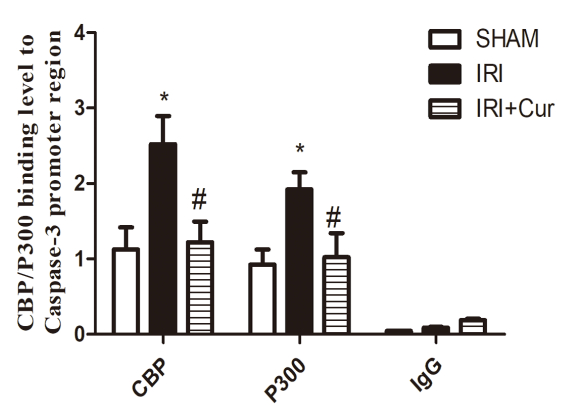

E

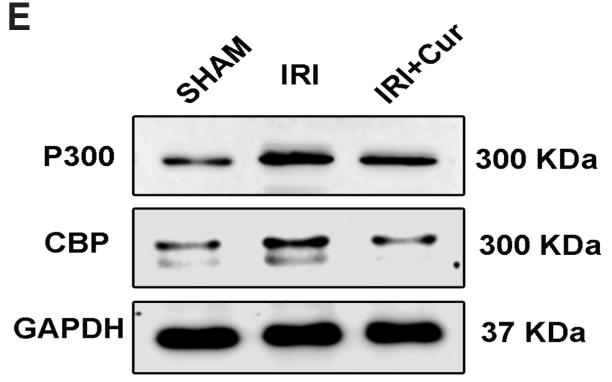

B

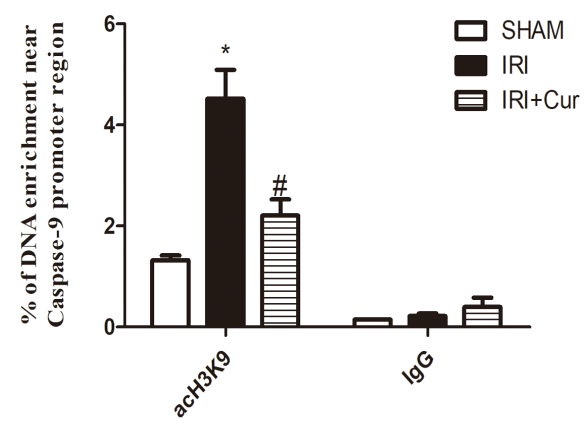

D

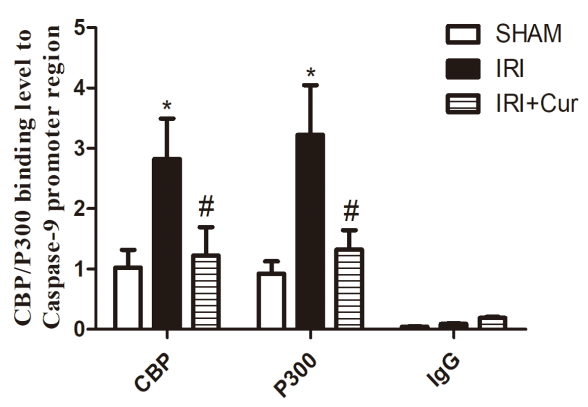

$\mathbf{F}$

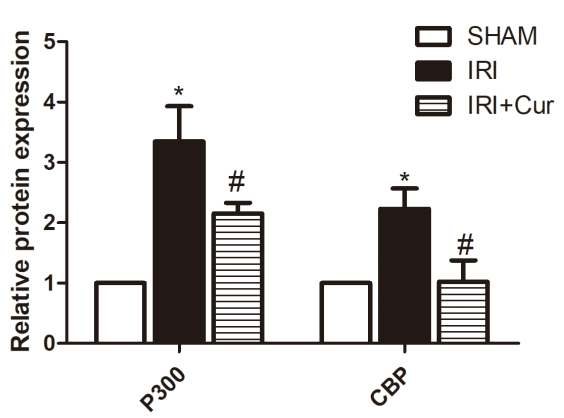

Fig. 5. Histone acetylation near the promoter region of caspae- 3 and caspase-9. ChiP assay was used to detect acH3K9 level in the upstream region of (A) caspase-3 (1,000 bp) and (B) caspase-9 $(1,000 \mathrm{bp})$. The enrichment of CREB-binding protein (CBP)/p300 in the promoter region of (C) caspase-3 and (D) caspase-9 in SHAM group, IRI group and IRI/Cur group. (E) The protein levels of p300 and CBP in above groups. (F) Quantification results of western blotting. Results are expressed as mean \pm SD. Statistical significance was determined by One-way ANOVA followed by Fisher's Least Significant Difference test. $\mathrm{n}=8$ per group. IRI, ischemia/reperfusion injury; Cur, curcumin. * $p<0.05$ vs. SHAM group; ${ }^{p} p<0.05$ vs. IRI group. caspase-3/-9 expression.

Curcumin is a specific inhibitor of HAT, especially for the inhibition of $\mathrm{CBP} / \mathrm{p} 300$ expression and HAT activity, thereby suppressing histone acetylation near target gene's promoter and repress gene expression [37]. Enhanced histone acetylation has been found in the damaged tissues and organs due to ischemia and reperfusion. In renal cortex, lysine acetylation has major presence in renal tubular cells, and significant increase in the fraction of acetylation-positive tubules could be seen in the kidney subjective to IRI [38]. IRI also enhances the amount of acH3K9 in whole cell lysates in the kidney and liver [39-41]. In addition, acH3K9 plays a critical role in cell apoptosis and enhances caspase expression [42]. For instance, enhanced $\mathrm{H} 3 \mathrm{~K} 9$ acetylation could promote caspase-3 and caspase-8 expressions in heart exposed to alcohol [43]. Thus, it's tempting to investigate whether enhanced caspase-3/-9 expression in IRI kidney were regulated via histone H3K9 acetylation. In the study, we confirmed the amount of acetylated histone $\mathrm{H} 3$ proteins was elevated in IRI kidney (Fig. 6B). We further found acH3K9 level near the promoter region of caspase-3/-9 and the expression of caspase-3/-9 proteins were both enhanced. By contrast, acH3K9 level and caspase-3/-9 expression decreased in IRI rats receiving curcumin treatment, suggesting that acH3K9 could serve as a critical role in IRI induced apoptosis.

HATs are epigenetic enzymes that are capable of acetylating the $\varepsilon$-amino group of specific lysine residues in histones. It is reported that renal IRI could induce $\mathrm{CBP} / \mathrm{p} 300$ expression, which could mediate acetylation of H3K9 [40,44]. Thus, we further explored whether $\mathrm{CBP} / \mathrm{p} 300$ were involved in acH3K9-induced caspase-3/-9 expression. In the study, we found the expression and activity of $\mathrm{CBP} / \mathrm{p} 300$ were enhanced in the kidneys of IRI rats. In addition, IRI promoted the binding of $\mathrm{CBP} / \mathrm{p} 300$ to the promoter region of caspase-3/-9. Our data showed that the binding level of $\mathrm{CBP} / \mathrm{p} 300$ to the promoter region of caspase-3/-9 was elevated in IRI rats. After curcumin treatment, inhibition was seen in CBP/ p300 expression and HAT activity as well as the binding level of $\mathrm{CBP} / 300$ to the promoter region of caspase-3/-9 in IRI rats. These 

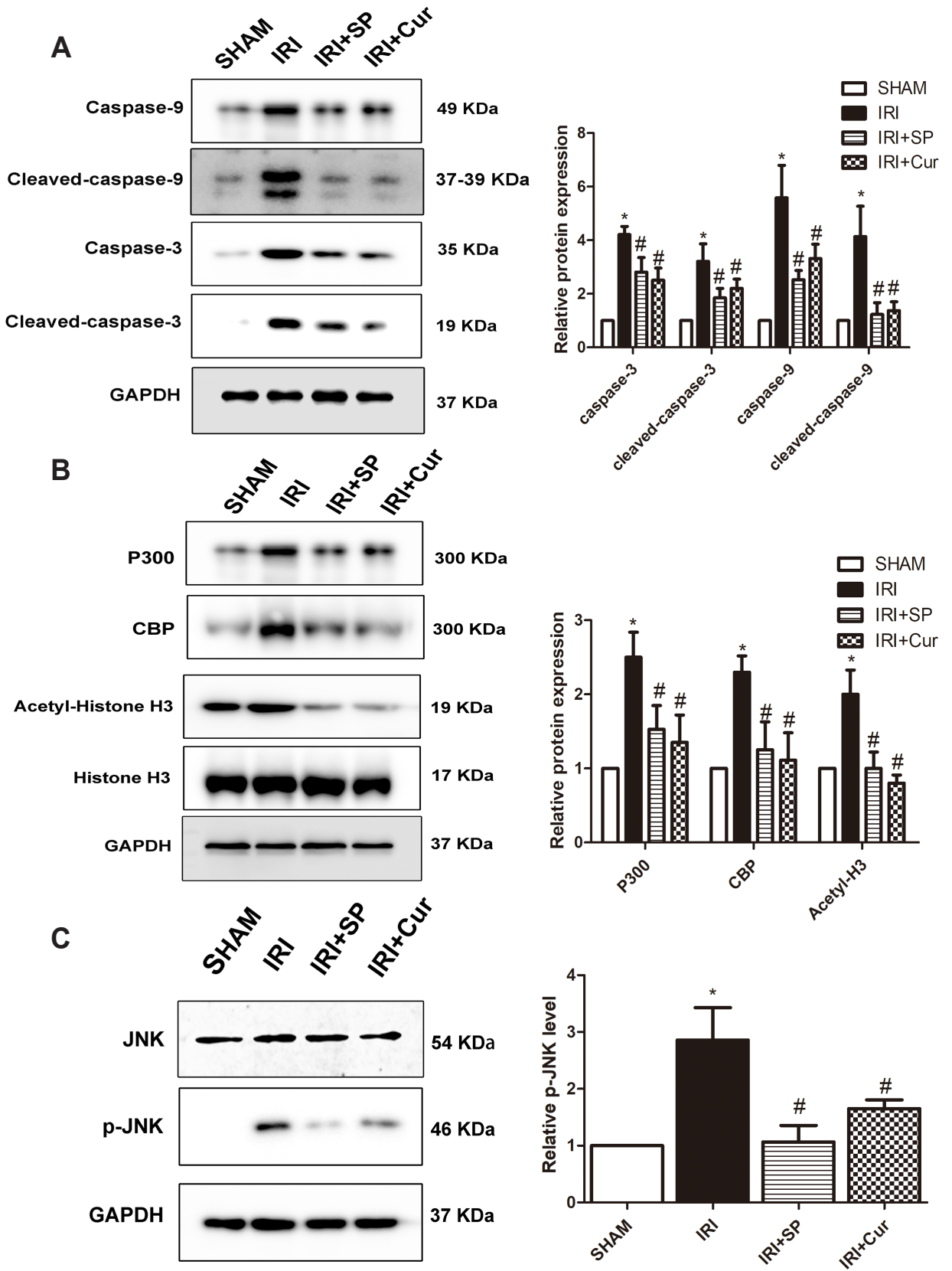

Fig. 6. c-Jun N-terminal kinase (JNK) and curcumin show similar effects on p300/CREB-binding protein (CBP) and caspase $3 / 9$ expression. (A) The protein levels of caspase-3/-9 and cleaved caspase-3/-9 in SHAM group, IRI group, IRI/ SP group and IRI/Cur group. (B) The level of histone acetylation related proteins including $\mathrm{p} 300 / \mathrm{CBP}$ and acetyl-histone $\mathrm{H} 3$ in above groups. (C) The protein level of phosphorylated JNK in above groups. Results are expressed as mean \pm SD. Statistical significance was determined by One-way ANOVA followed by Fisher's Least Significant Difference test. $\mathrm{n}=8$ per group. IRI, ischemia/reperfusion injury; SP, SP600125; Cur, curcumin. *p $<0.05$ vs. SHAM group; ${ }^{\mathrm{p}} \mathrm{p}<0.05$ vs. IRI group.

data indicate enhanced $\mathrm{CBP} / 300$ expression and activity would be held accountable for IRI induced caspase-3/-9 expression.

Accumulating evidence supports that activation of JNK signaling is a characteristic feature of human acute and chronic kidney diseases including renal IRI $[45,46]$. And JNK signaling plays a critical role in apoptosis in renal IRI [23]. In the study, we also found JNK signaling was activated in IRI rats and JNK inhibitor could suppress renal apoptosis and enhance renal function. It was reported that curcumin could inhibit JNK signaling to prevent renal fibrosis in diabetic nephropathy and protect cardiac cells against IRI [47,48]. Besides, JNK inhibitor could reduce H3 histone acetylation in peripheral sensory neurons [24]. Given these findings, we hypothesized that JNK signaling was involved in curcumin's regulation on caspase-3/-9 expression. In the study, we found the role of JNK inhibitor in regulation of caspase-3/-9 expression in IRI rats was very similar to that of curcumin. JNK inhibitor could inhibit IRI induced CBP/p300 expression and HAT activity, thereby resulting in decrease of $\mathrm{CBP} / \mathrm{p} 300$ binding to the promoter region of caspase-3/-9 and hence repressing the gene expression. These data revealed the role of JNK in epigenetic regulation of caspase-3/-9 expression. That JNK phosphorylation in kidneys of IRI rats receiving curcumin treatment was inhibited indicates curcumin could inhibit caspase-3/-9 expression via JNK/p300/CBP signaling pathway in IRI rats.

Collectively, the present study provides evidence that IRI induced renal apoptosis was regulated by histone acetylation via JNK pathway and curcumin possesses potential protective effects against renal apoptosis by inhibiting JNK and p300/CBP, as 
A

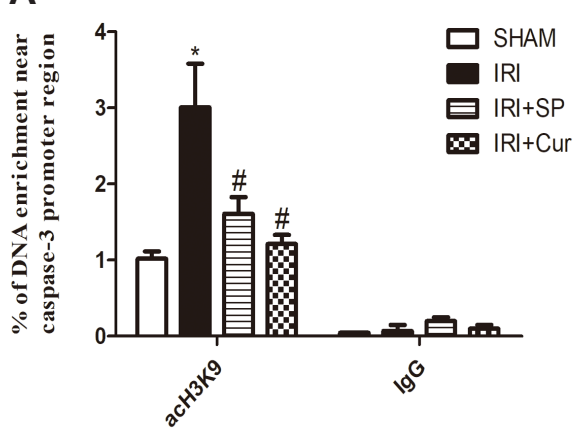

C

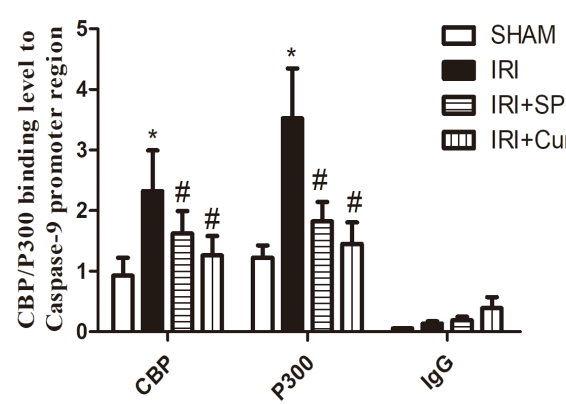

E

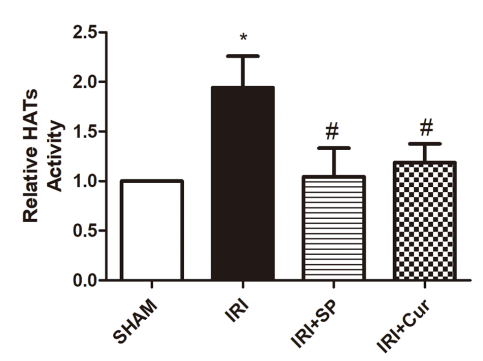

B

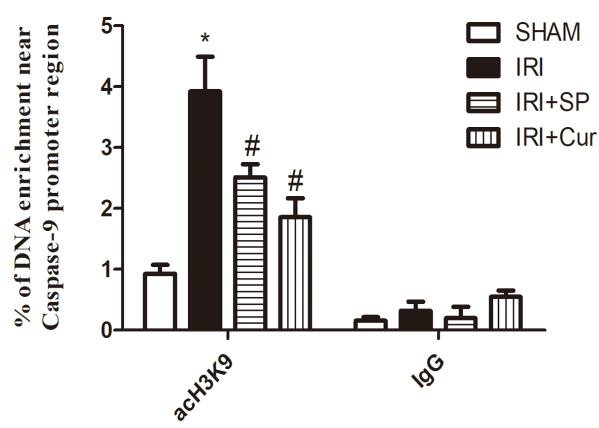

D

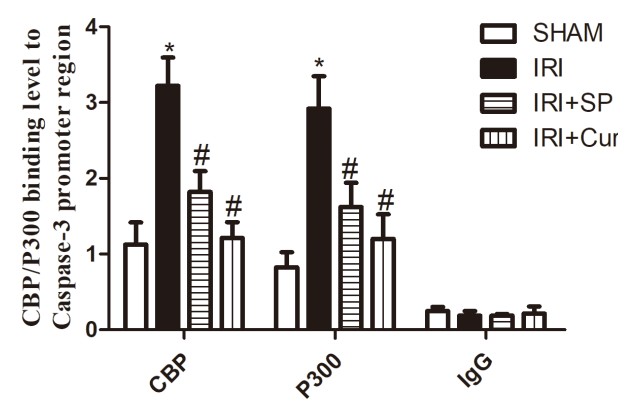

Fig. 7. c-Jun N-terminal kinase (JNK) and curcumin show similar effects on H3K9 acetylation near the promoter region of caspase-3/-9. The enrichment of acH3K9 in the promoter regions of (A) caspase-3 and (B) caspase- 9 in SHAM group, IRI group, IRI/SP group and IRI/Cur group. The binding level of CREB-binding protein (CBP)/p300 to the promoter region of (C) caspase-3 and (D) caspase- 9 in above groups. (E) histone acetyltransferase (HAT) activity in above groups. Results are expressed as mean \pm SD. Statistical significance was determined by One-way ANOVA followed by Fisher's Least Significant Difference test. $\mathrm{n}=8$ per group. IRI, ischemia/reperfusion injury; SP, SP600125; Cur, curcumin. ${ }^{*} \mathrm{p}<0.05$ vs. SHAM group; ${ }^{\mathrm{p}} \mathrm{p}<0.05$ vs. IRI group.

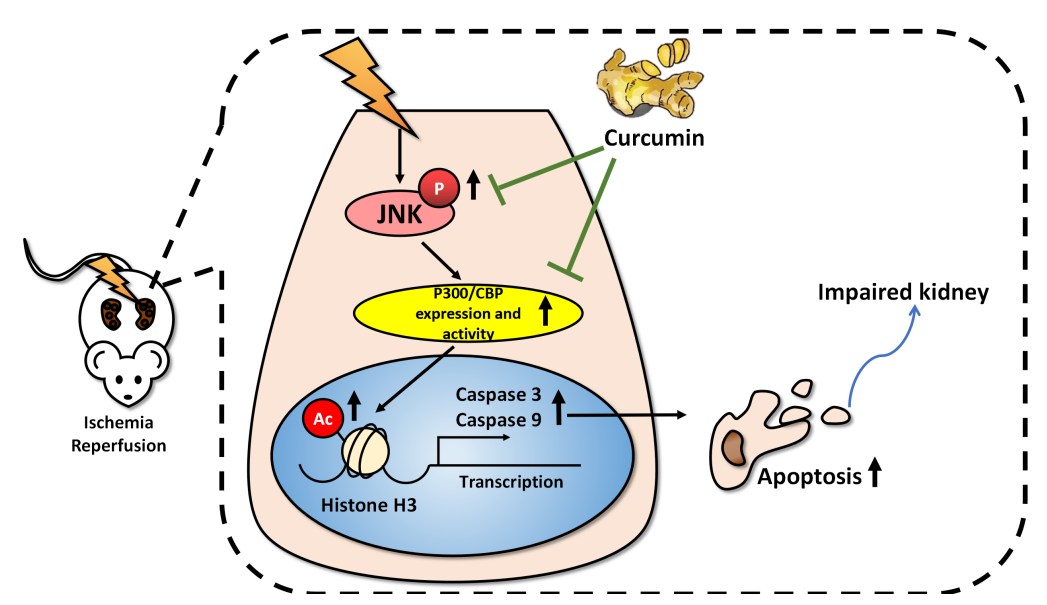

Fig. 8. Schematic illustration of the protective mechanism of curcumin against renal ischemia/reperfusion injury (IRI). The mechanism involves suppression on activation of c-Jun N-terminal kinase (JNK) pathway via epigenetic regulation of p300/CREB-binding protein (CBP)-mediated histone acetylation. In the IRI rat model, JNK activation promotes the elevation in acH3K9 level at caspase-3/-9 gene promoters, as well as the occupancy of $\mathrm{p} 300 / \mathrm{CBP}$ on the gene promoters. These events can enhance caspase-3/-9 expression, thereby resulting in renal cell apoptosis involved in IRI. Curcumin can attenuate these changes through its effects on JNK signaling and histone acetylation. 
presented in Fig. 8. Although the apparent lack of investigation of JNK or p300/CBP knockout or overexpression for further validation, our study reveals the evidence of renal apoptosis from an epigenetic point of view and provide us with a potential therapeutic path for renal IRI.

\section{ACKNOWLEDGEMENTS}

This study was supported by research grants from National Natural Science Foundation of China (Grant No. 81770619).

\section{CONFLICTS OF INTEREST}

The authors declare no conflicts of interest.

\section{SUPPLEMENTARY MATERIALS}

Supplementary data including three figures can be found with this article online at https://doi.org/10.4196/kjpp.2021.25.5.413.

\section{REFENRENCES}

1. Saat TC, van den Akker EK, IJzermans JN, Dor FJ, de Bruin RW. Improving the outcome of kidney transplantation by ameliorating renal ischemia reperfusion injury: lost in translation? J Transl Med. 2016;14:20.

2. Ditonno P, Impedovo SV, Palazzo S, Bettocchi C, Gesualdo L, Grandaliano G, Selvaggi FP, Battaglia M. Effects of ischemia-reperfusion injury in kidney transplantation: risk factors and early and longterm outcomes in a single center. Transplant Proc. 2013;45:26412644.

3. Rewa O, Bagshaw SM. Acute kidney injury-epidemiology, outcomes and economics. Nat Rev Nephrol. 2014;10:193-207.

4. Hoste EAJ, Kellum JA, Selby NM, Zarbock A, Palevsky PM, Bagshaw SM, Goldstein SL, Cerdá J, Chawla LS. Global epidemiology and outcomes of acute kidney injury. Nat Rev Nephrol. 2018;14:607625.

5. Kelly KJ. Distant effects of experimental renal ischemia/reperfusion injury. J Am Soc Nephrol. 2003;14:1549-1558.

6. Awad AS, El-Sharif AA. Curcumin immune-mediated and antiapoptotic mechanisms protect against renal ischemia/reperfusion and distant organ induced injuries. Int Immunopharmacol. 2011;11:992-996.

7. Bonegio R, Lieberthal W. Role of apoptosis in the pathogenesis of acute renal failure. Curr Opin Nephrol Hypertens. 2002;11:301-308.

8. Basile DP, Anderson MD, Sutton TA. Pathophysiology of acute kidney injury. Compr Physiol. 2012;2:1303-1353.

9. Zhao Y, Ding C, Xue W, Ding X, Zheng J, Gao Y, Xia X, Li S, Liu J, Han F, Zhu F, Tian P. Genome-wide DNA methylation analysis in renal ischemia reperfusion injury. Gene. 2017;610:32-43.

10. Fontecha-Barriuso M, Martin-Sanchez D, Ruiz-Andres O, Poveda J,
Sanchez-Niño MD, Valiño-Rivas L, Ruiz-Ortega M, Ortiz A, Sanz $\mathrm{AB}$. Targeting epigenetic DNA and histone modifications to treat kidney disease. Nephrol Dial Transplant. 2018;33:1875-1886.

11. Guo C, Dong G, Liang X, Dong Z. Epigenetic regulation in AKI and kidney repair: mechanisms and therapeutic implications. Nat ReV Nephrol. 2019;15:220-239.

12. Tessarz P, Kouzarides T. Histone core modifications regulating nucleosome structure and dynamics. Nat Rev Mol Cell Biol. 2014;15:703-708.

13. Shogren-Knaak M, Ishii H, Sun JM, Pazin MJ, Davie JR, Peterson CL. Histone H4-K16 acetylation controls chromatin structure and protein interactions. Science. 2006;311:844-847.

14. Tang J, Zhuang S. Histone acetylation and DNA methylation in ischemia/reperfusion injury. Clin Sci (Lond). 2019;133:597-609.

15. Bomsztyk K, Denisenko O. Epigenetic alterations in acute kidney injury. Semin Nephrol. 2013;33:327-340.

16. Audia JE, Campbell RM. Histone modifications and cancer. Cold Spring Harb Perspect Biol. 2016;8:a019521.

17. Bao L, Diao H, Dong N, Su X, Wang B, Mo Q, Yu H, Wang X, Chen C. Histone deacetylase inhibitor induces cell apoptosis and cycle arrest in lung cancer cells via mitochondrial injury and p53 upacetylation. Cell Biol Toxicol. 2016;32:469-482.

18. Li D, Zeng Z. Epigenetic regulation of histone $\mathrm{H} 3$ in the process of hepatocellular tumorigenesis. Biosci Rep. 2019;39:BSR20191815.

19. Ammon HP, Wahl MA. Pharmacology of Curcuma longa. Planta Med. 1991;57:1-7.

20. Marcu MG, Jung YJ, Lee S, Chung EJ, Lee MJ, Trepel J, Neckers L. Curcumin is an inhibitor of p300 histone acetylatransferase. Med Chem. 2006;2:169-174.

21. Wang X, Muhammad I, Sun X, Han M, Hamid S, Zhang X. Protective role of curcumin in ameliorating AFB1-induced apoptosis via mitochondrial pathway in liver cells. Mol Biol Rep. 2018;45:881-891.

22. Yu W, Zha W, Ke Z, Min Q, Li C, Sun H, Liu C. Curcumin protects neonatal rat cardiomyocytes against high glucose-induced apoptosis via PI3K/Akt signalling pathway. J Diabetes Res. 2016;2016:4158591.

23. Kunduzova OR, Bianchi P, Pizzinat N, Escourrou G, Seguelas MH, Parini A, Cambon C. Regulation of JNK/ERK activation, cell apoptosis, and tissue regeneration by monoamine oxidases after renal ischemia-reperfusion. FASEB J. 2002;16:1129-1131.

24. Wu J, Zhang X, Nauta HJ, Lin Q, Li J, Fang L. JNK1 regulates histone acetylation in trigeminal neurons following chemical stimulation. Biochem Biophys Res Commun. 2008;376:781-786.

25. Bayrak O, Uz E, Bayrak R, Turgut F, Atmaca AF, Sahin S, Yildirim ME, Kaya A, Cimentepe E, Akcay A. Curcumin protects against ischemia/reperfusion injury in rat kidneys. World J Urol. 2008;26:285-291.

26. Wu J, Pan X, Fu H, Zheng Y, Dai Y, Yin Y, Chen Q, Hao Q, Bao D, Hou D. Effect of curcumin on glycerol-induced acute kidney injury in rats. Sci Rep. 2017;7:10114.

27. Xu YF, Liu M, Peng B, Che JP, Zhang HM, Yan Y, Wang GC, Wu YC, Zheng JH. Protective effects of SP600125 on renal ischemiareperfusion injury in rats. J Surg Res. 2011;169:e77-e84.

28. Zhao W, Wu X, Wang Z, Pan B, Liu L, Liu L, Huang X, Tian J. Epigenetic regulation of phosphodiesterase $4 \mathrm{~d}$ in restrictive cardiomyopathy mice with cTnI mutations. Sci China Life Sci. 2020;63:563570.

29. Zhou W, Jiang D, Tian J, Liu L, Lu T, Huang X, Sun H. Acetylation 
of $\mathrm{H} 3 \mathrm{~K} 4, \mathrm{H} 3 \mathrm{~K}$, and $\mathrm{H} 3 \mathrm{~K} 27$ mediated by $\mathrm{p} 300$ regulates the expression of GATA4 in cardiocytes. Genes Dis. 2018;6:318-325.

30. Gupta SC, Patchva S, Aggarwal BB. Therapeutic roles of curcumin: lessons learned from clinical trials. AAPS J. 2013;15:195-218.

31. Hasan ST, Zingg JM, Kwan P, Noble T, Smith D, Meydani M. Curcumin modulation of high fat diet-induced atherosclerosis and steatohepatosis in LDL receptor deficient mice. Atherosclerosis. 2014;232:40-51.

32. Cekmen M, Ilbey YO, Ozbek E, Simsek A, Somay A, Ersoz C. Curcumin prevents oxidative renal damage induced by acetaminophen in rats. Food Chem Toxicol. 2009;47:1480-1484.

33. Zhang J, Tang L, Li GS, Wang J. The anti-inflammatory effects of curcumin on renal ischemia-reperfusion injury in rats. Ren Fail. 2018;40:680-686.

34. Ibrahim SG, El-Emam SZ, Mohamed EA, Abd Ellah MF. Dimethyl fumarate and curcumin attenuate hepatic ischemia/reperfusion injury via $\mathrm{Nrf} 2 / \mathrm{HO}-1$ activation and anti-inflammatory properties. Int Immunopharmacol. 2020;80:106131.

35. Yeh CH, Chen TP, Wu YC, Lin YM, Jing Lin P. Inhibition of NFkap$\mathrm{paB}$ activation with curcumin attenuates plasma inflammatory cytokines surge and cardiomyocytic apoptosis following cardiac ischemia/reperfusion. J Surg Res. 2005;125:109-116.

36. Mokhtari-Zaer A, Marefati N, Atkin SL, Butler AE, Sahebkar A. The protective role of curcumin in myocardial ischemia-reperfusion injury. J Cell Physiol. 2018;234:214-222.

37. Boyanapalli SS, Kong AT. "Curcumin, the King of Spices": epigenetic regulatory mechanisms in the prevention of cancer, neurological, and inflammatory diseases. Curr Pharmacol Rep. 2015;1:129-139.

38. Jankauskas SS, Pevzner IB, Andrianova NV, Zorova LD, Popkov VA, Silachev DN, Kolosova NG, Plotnikov EY, Zorov DB. The ageassociated loss of ischemic preconditioning in the kidney is accompanied by mitochondrial dysfunction, increased protein acetylation and decreased autophagy. Sci Rep. 2017;7:44430.

39. Zager RA, Johnson AC, Becker K. Acute unilateral ischemic renal injury induces progressive renal inflammation, lipid accumulation, histone modification, and "end-stage" kidney disease. Am J Physiol Renal Physiol. 2011;301:F1334-F1345.
40. Bomsztyk K, Flanagin S, Mar D, Mikula M, Johnson A, Zager R, Denisenko O. Synchronous recruitment of epigenetic modifiers to endotoxin synergistically activated Tnf- $\alpha$ gene in acute kidney injury. PLoS One. 2013;8:e70322.

41. Evankovich J, Cho SW, Zhang R, Cardinal J, Dhupar R, Zhang L, Klune JR, Zlotnicki J, Billiar T, Tsung A. High mobility group box 1 release from hepatocytes during ischemia and reperfusion injury is mediated by decreased histone deacetylase activity. J Biol Chem. 2010;285:39888-39897.

42. Li J, Chen P, Sinogeeva N, Gorospe M, Wersto RP, Chrest FJ, Barnes J, Liu Y. Arsenic trioxide promotes histone $\mathrm{H} 3$ phosphoacetylation at the chromatin of CASPASE-10 in acute promyelocytic leukemia cells. J Biol Chem. 2002;277:49504-49510.

43. Yan X, Pan B, Lv T, Liu L, Zhu J, Shen W, Huang X, Tian J. Inhibition of histone acetylation by curcumin reduces alcohol-induced fetal cardiac apoptosis. J Biomed Sci. 2017;24:1.

44. Peng C, Zhang W, Zhao W, Zhu J, Huang X, Tian J. Alcohol-induced histone $\mathrm{H} 3 \mathrm{~K} 9$ hyperacetylation and cardiac hypertrophy are reversed by a histone acetylases inhibitor anacardic acid in developing murine hearts. Biochimie. 2015;113:1-9.

45. Tesch GH, Ma FY, Nikolic-Paterson DJ. ASK1: a new therapeutic target for kidney disease. Am J Physiol Renal Physiol. 2016;311:F373F381.

46. Kanellis J, Ma FY, Kandane-Rathnayake R, Dowling JP, Polkinghorne KR, Bennett BL, Friedman GC, Nikolic-Paterson DJ. JNK signalling in human and experimental renal ischaemia/reperfusion injury. Nephrol Dial Transplant. 2010;25:2898-2908.

47. Wang Y, Wang Y, Luo M, Wu H, Kong L, Xin Y, Cui W, Zhao Y, Wang J, Liang G, Miao L, Cai L. Novel curcumin analog C66 prevents diabetic nephropathy via JNK pathway with the involvement of p300/CBP-mediated histone acetylation. Biochim Biophys Acta. 2015;1852:34-46.

48. Fiorillo C, Becatti M, Pensalfini A, Cecchi C, Lanzilao L, Donzelli G, Nassi N, Giannini L, Borchi E, Nassi P. Curcumin protects cardiac cells against ischemia-reperfusion injury: effects on oxidative stress, NF-kappaB, and JNK pathways. Free Radic Biol Med. 2008;45:839846. 